\title{
ELABORACIÓN Y EVALUACIÓN DE POLVOS PARA BEBIDAS INSTÁNTANEAS A BASE DE HARINA EXTRUDIDA DE ÑME (Dioscorea alata)
}

\section{ELABORATION AND EVALUATION OF POWDERS FOR INSTANT DRINKS BASED UPON EXTRUDED FLOUR OF YAM (Dioscorea alata)}

\author{
Emperatriz Pacheco-Delahaye, Nora Techeira, Auris D. García \\ Laboratorio de Bioquímica de Alimentos del Instituto de Química y Tecnología. Facultad de Agronomía. \\ Universidad Central de Venezuela, Maracay, Estado Aragua, Venezuela.
}

\begin{abstract}
The tubercles of Dioscorea alata which belongs to the Dioscoraceae family are rhizomes, which are cultivated in the tropical and subtropical regions of the world; representing an energetic food source for these populations due to its high contents of starch. In order to increase and diversify its consumption, it was proposed to study the addition of extruded yam flour for the elaboration of a powder mixture for making vanilla-flavored instant drinks. Two formulations were made based on $20 \%$ and $40 \%$ of extruded yam flour; they were evaluated in function to their chemical composition, physical characteristics (water activity, color, $\mathrm{pH}$ and viscosity) and sensorial properties. The results indicated that the addition of the extruded yam flour allowed to increase the protein and dietary fiber contents, with values of water activity, $\mathrm{pH}$ and viscosity similar to those of the commercial product that was taken as a reference. The sensorial evaluation resulted of good approval for the studied formulations. In conclusion, the formulation based upon $20 \%$ of extruded yam flour, allowed obtaining a product with chemical, physical and sensorial properties comparables to the commercial product; but with antioxidant properties ascribed to some polyphenols originated from the extruded yam flour.
\end{abstract}

Key words: Dioscorea alata, flour, powder, instant drink.

Este trabajo fue recibido el 23 de Enero de 2008 y aceptado para ser publicado el 10 de Noviembre de 2008.

\section{INTRODUCCIÓN}

Las raíces y tubérculos tropicales son considerados alimentos básicos en la dieta de los pobladores de América Latina, África y Asia; y a pesar de que un gran número de estos cultivos se podrían desarrollar a nivel mundial, sólo cinco especies ocupan el 99\% del total de la producción papa (Solanum tuberosum), yuca (Manihot esculenta), batata (Ipomoea batatas), ñame (Dioscorea spp.) y ocumo (Colocassia, Cytosperma, Xanthosoma spp.) (1).

En Venezuela, la mayoría de los cultivos de raíces y tubérculos, entre ellos la batata, ocumo, apio (Arracacia xanthorrhiza) y ñame, se manejan en el ámbito de pequeños sembradíos y se comercializan principalmente a nivel nacional, teniendo un valor comercial bastante escaso en los canales internacionales, por no incursionar estos mercados. En general, estos rubros son perecederos y se caracterizan por tener un elevado contenido de humedad, resultando como consecuencia pérdidas postcosecha que alcanzan hasta un $30 \%$ a nivel mundial (2).

El ñame es un cultivo que pertenece a la familia Dioscoreaceae, recomendado como alimento energético debido a que presenta un alto contenido de almidón (70 a $80 \%$ de su peso en materia seca), y una concentración de proteínas del $12 \%$, que se considera superior a la determinada para otras raíces y tubérculos tropicales, mostrando un buen balance de aminoácidos esenciales (3). Además, contiene fibra dietaria (7 a 8\%), compuestos antioxidantes, vitaminas y minerales, tales como ácido ascórbico $(30 \mu \mathrm{g} / \mathrm{g})$, calcio $(140 \mu \mathrm{g} / \mathrm{g})$ y fósforo (430 
$\mu \mathrm{g} / \mathrm{g})$, que incrementan su valor nutricional $(3,4)$.

Por otro lado, el ñame es muy utilizado en la medicina tradicional china, ya que los rizomas de ñame se utilizan para el tratamiento de la indigestión, anorexia, diarrea y diabetes. Además, se presume que tiene un efecto hipoglicémico y promueve la salud de las mujeres de la tercera edad. Se utiliza como fuente de diosgenina (precursor de progesterona), cortisona y otros esteroides médicamente importantes $(5,6)$.

En vista de que los tubérculos de ñame, así como sus extractos y harinas, contienen compuestos antioxidantes, son altamente recomendados para mitigar el daño y las enfermedades causadas por compuestos oxidativos, entre las cuales se encuentran ciertos procesos degenerativos o patológicos como el envejecimiento, el cáncer, las enfermedades coronarias y el mal de Alzheimer. Entre los compuestos antioxidantes más abundantes se encuentran algunos polifenoles (catequinas, ácido clorogénico y antocianinas); fitoquímicos (ácido ascórbico, tocoferol) y pigmentos $(7,8)$.

Ciertos estudios han demostrado el poder antioxidante del ñame; al respecto Bhandari y Kawabata (9), determinaron que extractos acuosos obtenidos a partir de dicho tubérculo, tenían un elevado poder reductor y una gran actividad quelante de iones ferrosos. Por otro lado, Hsu y col. (10), desarrollaron un producto de panadería mediante la sustitución de $25 \%$ de harina de trigo por harina de $D$. purpurea, una de las principales variedades de ñame cultivadas en Taiwán.

En cuanto al almidón de ñame, al igual que el resto de los almidones de raíces y tubérculos, presenta bajas temperaturas de gelatinización $\left(65\right.$ a $\left.75^{\circ} \mathrm{C}\right)$, y sus gránulos se hinchan rápido y uniformemente, exhibiendo una alta viscosidad y formando pastas de alta claridad comparado con los almidones de cereales. A diferencia de los almidones de la mayoría de las raíces y tubérculos, el almidón de ñame es capaz de mantener los valores de viscosidad estables a altas temperaturas y bajos valores de $\mathrm{pH}$. Por lo tanto, los almidones de ñame podrían considerarse como fuente alternativa de almidón comercial, y como un ingrediente idóneo en la elaboración de sopas tipo instantáneas; mientras que los elevados valores de viscosidad desarrollados en las suspensiones de harina sugieren su utilización como agentes espesantes $(11,12)$.

La extrusión es el proceso por medio del cual los materiales amiláceos y proteínicos de carácter expansible, son sometidos a cocción y plastificación, en combinación con ciertos factores, tales como humedad, presión, temperatura y energía mecánica; y se caracteriza por su versatilidad, que ha permitido utilizarlo en la elaboración de diversos productos alimenticios, entre los cuales se encuentran los cereales para desayuno, bocados y las harinas precocidas $(13,14)$.

La utilización de harinas extrudidas para la obtención de ciertos productos alimenticios resulta ventajosa, puesto que el proceso de extrusión conlleva a la pregelatinización de los gránulos de almidón, provocando la pérdida del orden molecular y la completa degradación de los polímeros con la formación de fragmentos altamente solubles. Por lo tanto, las suspensiones de harinas precocidas por extrusión son capaces de incrementar rápidamente su viscosidad, presentando una baja tendencia a la formación de grumos, ya que los gránulos de almidón han sido modificados, y muestran un gran poder de hinchamiento en frío y en caliente, siendo altamente recomendados en la elaboración de productos alimenticios instantáneos (15).

La elaboración de bebidas a base de harinas de raíces y tubérculos, resultan de interés ya que éstas proporcionan características espesantes, estabilizantes, consistencia del fluido viscoelástico, claridad de las pastas $\mathrm{y}$ estabilidad en un amplio intervalo de $\mathrm{pH}$ e incluso en agitación mecánica y en ciclos de congelación /descongelación, siendo por ello adecuadas para la obtención de productos en polvo de rápida preparación (16).

En base a lo expuesto, se planteó como objetivo del presente trabajo formular y elaborar una mezcla en polvo para preparar bebidas instantáneas saborizada con vainilla a base de harina extrudida de ñame y la incorporación de leche en polvo completa y sacarosa, evaluando las características químicas, físicas y sensoriales. Propuesta que fue considerada, dado que estos productos pertenecen a la variedad de alimentos deshidratados más representativos y de mayor consumo en el mercado como alimentos instantáneos, que en muchos casos solo requieren la adición de agua para su preparación. Siendo su impacto social positivo frente al consumidor, principalmente en aquellas personas que disponen de poco tiempo y requieren elaborar de forma rápida sus alimentos (17).

\section{SUJETOS Y MÉTODOS Elaboración de la mezcla en polvo para preparar bebidas instantáneas}

La harina extrudida de ñame (Dioscorea alata) se obtuvo a partir de los tubérculos de la variedad blanca, los que fueron lavados, pelados, troceados e inmersos en una solución de acido cítrico al $0,1 \%$ y secado por convección a $80^{\circ} \mathrm{C}$ por 4 horas, que luego fueron sometidos a un proceso de molienda en un molino de martillo (marca Thomas-Wiley, Modelo 4). Obtenida la harina se ajustó su contenido de humedad a $15 \pm 1 \%$, para ser sometida al proceso de extrusión en un equipo extrusor 
de laboratorio de tipo monotornillo, marca Brabender, modelo AEV.330, a una velocidad de alimentación de 60 rpm, velocidad de tornillo de $120 \mathrm{rpm}$, y temperaturas de extrusión de $75^{\circ} \mathrm{C}$ en la primera zona y de $180^{\circ} \mathrm{C}$ en la tercera zona. Los "collets" obtenidos en el proceso de extrusión fueron secados a $90^{\circ} \mathrm{C}$ por 30 minutos y sometidos a un proceso de molienda hasta alcanzar un tamaño de partícula equivalente a 60 mesh, siendo la harina extrudida de ñame empacada al vacío y mantenida a temperatura ambiente temporalmente hasta su uso (figura 1).

La formulación de la mezcla en polvo para preparar la bebida instantánea se basó en la obtención de un producto listo para el consumo, con el solo añadido de agua. Para ello se formuló una mezcla a base de la harina extrudida de ñame, leche entera en polvo, azúcar en polvo como edulcorante y para saborizar se incorporó el polvo de vainillina (figura 1). Para preparar la mezcla, se plantearon dos formulaciones distintas, una donde la harina extrudida de ñame se incorporó en un $40 \%$ (F1), $y$ otra en un $20 \%$ (F2) (tabla 1). Ambas formulaciones se compararon entre sí y con un producto comercial análogo $(\mathrm{Fc})$ de amplio consumo en Venezuela.

\section{Análisis químicos}

Se determinó la composición química aplicando los métodos de la AOAC (18) para la determinación del contenido de humedad (método $\mathrm{n}^{\circ} 925.09$ ), cenizas (método $\mathrm{n}^{\circ} 923.03$ ), proteína cruda $(\mathrm{N} \times 6,25)$ (método ${ }^{\circ}$ 979.09), grasa cruda (método $\mathrm{n}^{\circ}$ 920.39), fibra dietética (método enzimático-gravimétrico $\mathrm{n}^{\circ}$ 985.29), azúcares reductores (método ${ }^{\circ}$ 925.36), almidón por el método de

\section{FIGURA 1}

\section{Esquema tecnológico para obtener la harina extrudida de ñame y la formulación de mezcla en polvo para preparar bebidas instantáneas a base de esta harina}

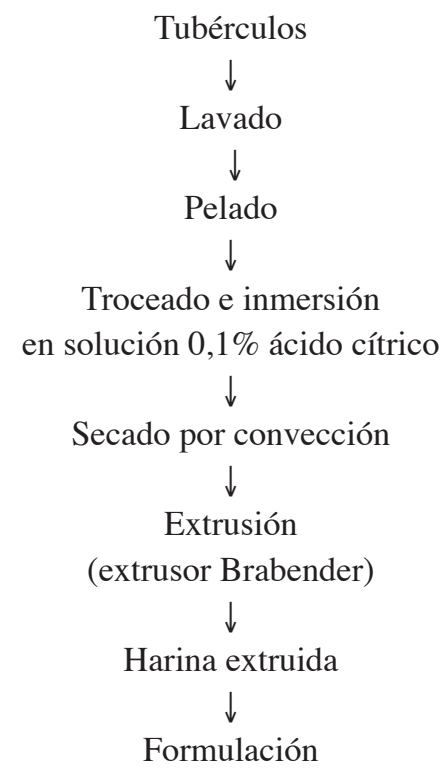

Harina extruida de ñame, leche entera en polvo + azúcar en polvo + vainillina en polvo

$\downarrow$

Homogenización

$\downarrow$

Empacado al vacío

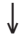

Alamacenamiento a temperatura ambiente 
Holm (19) y polifenoles totales por el método de Kaluza y col. (20), utilizando el reactivo Folin-Ciocalten. Todas las determinaciones se hicieron por triplicado.

\section{Análisis físicos}

Se efectuaron las determinaciones del contenido de actividad de agua, mediante un equipo psicrométrico Equoi Aqualab Decagon cx-2; el color medido como el valor de luminosidad, de acuerdo a la metodología descrita por Hunter Lab, el pH se realizo haciendo uso de un pHmetro marca Orion y la viscosidad aparente a la temperatura de $25^{\circ} \mathrm{C}$ en las preparaciones reconstituidas, utilizando el viscosímetro Brookfield (Modelo LVF, serial 63127, Brookfield Laboratories Inc., Stoughton, MA, USA). Todas las determinaciones se realizaron por triplicado.

\section{Evaluación sensorial}

La evaluación sensorial de las dos formulaciones de las mezclas en polvos para preparar las bebidas en estudio y la del producto comercial análogo, se realizaron con un panel no entrenado de 50 estudiantes de la Facultad de Agronomía de la Universidad Central de Venezuela, de ambos sexos y con edades comprendidas entre los 20 y 26 años, a los cuales se les suministró el alimento reconstituidos en agua y una planilla de evaluación, para que emitieran su opinión acerca de los atributos color, sabor, aroma y consistencia, haciendo uso de una escala hedónica, que permitió a los panelistas manifestar su grado de aceptación por cada uno de los productos.

\section{Análisis Estadístico}

Los resultados obtenidos del análisis químico y físico se analizaron mediante un análisis de varianza de una sola vía, para determinar la existencia de dife- rencias estadísticamente significativas entre muestras. Mientras para la evaluación sensorial, se analizó el nivel de aceptación de las muestras en estudio, utilizando el análisis de comparación múltiple entre rangos, descrito por Pedrero y Pangborn (21). Estos análisis se hicieron con el uso del programa computarizado "Statistix" para soporte técnico de Windows 2000.

\section{RESULTADOS Y DISCUSIÓN}

En la tabla 2 se presentan los resultados correspondientes a la composición química de las formulaciones en estudio (F1 y F2), del producto comercial $(\mathrm{Fc})$ y de la harina extrudida de ñame.

En la composición química de la harina de ñame se destaca su elevado contenido de almidón $(80,1 \%)$ y de fibra dietética $(7,6 \%)$, por lo cual puede ser considerada como un producto alimenticio con un gran valor energético y con una gran capacidad para prevenir la aparición de enfermedades crónicas, debido a que ciertos estudios han demostrado que el consumo de fibra dietética se correlaciona con una baja incidencia de diabetes, hipertensión arterial y obesidad (Wittig de Penna y cols.) (22). Estos resultados son comparables a lo reportado para otras harinas de ñame y para harinas provenientes de diferentes raíces y tubérculos (Blanco y cols.) (23), no obstante Freitas y col. (24), publicaron valores de $88 \%$ de almidón en harina de ñame del Brasil. En cuanto al contenido de cenizas $(2,90 \%)$ y proteínas $(6,75 \%)$, estos valores fueron superiores a los determinados por Leonel y cols. (25), que obtuvieron $2,21 \%$ de cenizas y $5,81 \%$ de proteínas.

El análisis de la composición química realizado a las diferentes formulaciones de bebidas instantáneas, reportó valores de proteína (F1: 15,12\%; F2: 19,10\%) y grasa $(8,60 \%$ para F1 y $10,8 \%$ para F2), superiores a los determinados para el producto comercial $(10,2 \%$ de

\section{TABLA 1}

\section{Formulación de las mezclas en polvo para preparar bebidas instantáneas a base de la harina extrudida de ñame}

Ingredientes

Harina extrudida de ñame

Leche entera en polvo

Azúcar en polvo

Vainillina en polvo
Proporción (\%)

Formulaciones planteadas

F1

40,00

20,00

34,00

54,02

25,82

25,80

0,18

0,18 
proteínas y $8,42 \%$ de grasa). Las diferencias existentes para el contenido de proteínas entre la muestra comercial y las formulaciones en estudio, pudieran atribuirse a la incorporación de la harina extrudida de ñame y a la leche entera, ya que la bebida instantánea con el mayor porcentaje de leche (F2) fue la que presentó los valores más altos de proteína. No obstante, las diferencias para el contenido de grasa, respecto a la muestra comercial, estarían determinadas principalmente por la utilización de leche en polvo entera en la formulación de las bebidas, más que por la incorporación de la harina extrudida de ñame, ya que ésta presenta solamente un $0,28 \%$ de grasa cruda.

$\mathrm{Al}$ analizar el contenido de cenizas (3,08\% para F1 y $2,75 \%$ para F2) y de fibra dietética (F1 con 3,90\% y F2 con $3,40 \%$ ), se observa que los valores obtenidos también fueron superiores a los determinados en el producto comercial $(2,11 \%$ y $1,70 \%$, respectivamente), debido posiblemente a la incorporación de la harina extrudida de ñame en la formulación de las bebidas instantáneas, ya que ésta se caracteriza por su elevado contenido de fibra dietética y de componentes minerales.

En relación al contenido de almidón, éste fue superior en la bebida F1, preparada a partir de $40 \%$ de harina de ñame $(39,6 \%)$, debido al mayor porcentaje de sustitución, ya que la bebida F2, con un $20 \%$ de harina de ñame, tenía un valor más bajo, equivalente a $18,20 \%$. Asimismo, al analizar los valores obtenidos para la concentración de polifenoles totales, se observa que existen diferencias significativas, ya que la bebida $\mathrm{F} 1$, presenta un valor superior $(34,20 \mu \mathrm{g} / \mathrm{g})$ al obtenido para la formulación F2 (31,20 $\mu \mathrm{g} / \mathrm{g})$; no obstante, ambas pueden ser consideradas como productos alimenticios con antioxidantes, debido al contenido de compuestos fenólicos. En la bebida comercial no se detectaron polifenoles.

Los resultados obtenidos en la composición química de las mezclas en polvo para preparar las bebidas instantáneas en estudio, y específicamente aquellos referidos al contenido de cenizas, proteína y grasa cruda, fueron similares a los reportados por Arcila y Mendoza (26) para una bebida instantánea a base de harina de semillas de amaranto, maíz y arroz; pero superiores a los determinados por Pacheco y cols. (27), quienes al elaborar polvos para bebidas instantáneas a base de papaya, plátano verde y salvado de arroz, encontraron valores de cenizas entre 2,56 y $3,80 \%$, de proteínas entre 5,7 y $7,25 \%$ y de grasa entre 0,70 y $1,56 \%$.

En la tabla 3 se muestran los valores correspondientes a las características físicas de las mezclas en polvo para preparar las bebidas instantáneas en estudio y de la harina extrudida de ñame. La medida física de la

\section{TABLA 2}

\section{Composición química de las mezclas en polvo para preparar bebidas instantáneas a base de la harina extrudida de ñame y de la harina extruida de ñame.}

\begin{tabular}{|c|c|c|c|c|}
\hline $\begin{array}{l}\text { Composición química } \\
\text { (g. / } 100 \text { g) }\end{array}$ & F1 & F2 & $\mathbf{F c} *$ & HN \\
\hline Humedad & $7,00 \pm 0,50^{\mathrm{a}}$ & $6,80 \pm 0,40^{\mathrm{a}}$ & $7,50 \pm 0,20^{\mathrm{a}}$ & $5,40 \pm 0,25^{b}$ \\
\hline Cenizas & $3,08 \pm 0,01^{\mathrm{a}}$ & $2,75 \pm 0,02^{\mathrm{a}}$ & $2,11 \pm 0,01^{\mathrm{b}}$ & $2,90 \pm 0,12^{\mathrm{a}}$ \\
\hline Proteína cruda & $5,12 \pm 0,08^{b}$ & $19,10 \pm 0,08^{\mathrm{a}}$ & $10,20 \pm 0,10^{c}$ & $6,75 \pm 0,02^{\mathrm{d}}$ \\
\hline Grasa cruda & $8,60 \pm 0,02^{\mathrm{b}}$ & $10,80 \pm 0,05^{\mathrm{a}}$ & $8,42 \pm 0,04^{\mathrm{b}}$ & $0,28 \pm 0,00^{\mathrm{c}}$ \\
\hline Fibra dietética & $3,90 \pm 0,01^{\mathrm{b}}$ & $3,40 \pm 0,01^{\mathrm{c}}$ & $1,70 \pm 0,01^{\mathrm{d}}$ & $7,60 \pm 0,01^{\mathrm{a}}$ \\
\hline Almidón & $39,60 \pm 0,18^{b}$ & $18,20 \pm 0,19^{c}$ & $11,0 \pm 0,40^{\mathrm{d}}$ & $80,10 \pm 0,39^{a}$ \\
\hline Azúcares reductores & $26,70 \pm 0,50^{\mathrm{b}}$ & $26,30 \pm 0,55^{\mathrm{b}}$ & $30,0 \pm 0,70^{\mathrm{a}}$ & $0,35 \pm 0,39^{\mathrm{c}}$ \\
\hline $\begin{array}{l}\text { Polifenoles totales } \\
\text { (equivalentes a ácido gálico, } \\
\text { expresados en } \mu \mathrm{g} / \mathrm{g} \text { ) }\end{array}$ & $34,20 \pm 0,01^{\mathrm{b}}$ & $31,20 \pm 0,01^{\mathrm{b}}$ & $0,001 \pm 0,00^{c}$ & $61,20 \pm 0,02^{\mathrm{a}}$ \\
\hline $\begin{array}{l}\text { Los valores se presentan como la } \\
\text { F1: } 40 \% \text { harina extrudida de ñam } \\
\text { F2: } 20 \% \text { harina extrudida de ñam } \\
\text { Fc: producto análogo comercial } \\
\text { HN: harina extrudida de ñame. }\end{array}$ & $\begin{array}{l}\text { desviación est } \\
6 \text { leche entera } \\
6 \text { leche entera }\end{array}$ & $\begin{array}{l}5,82 \% \text { azúcar e } \\
5,80 \% \text { azúcar er }\end{array}$ & $\begin{array}{l}18 \% \text { vainillin } \\
8 \% \text { vainillina }\end{array}$ & \\
\hline
\end{tabular}


actividad de agua (aw) no mostró diferencias estadísticamente significativas entre las muestras $(0,42$ para $\mathrm{F} 1$ y 0,41 para F2), lo cual confiere una gran estabilidad durante el almacenamiento, ya que valores superiores a 0,5-0,6, determinan la aparición de reacciones químicas, enzimáticas y de crecimiento microbiano que conllevan al deterioro del producto (28). Estos resultados coinciden con los reportados por Pacheco y cols. (27), quienes al evaluar formulaciones de las mezclas en polvos para preparar las bebidas a base de papaya, plátano verde y salvado de arroz, obtuvieron valores de 0,4 y 0,43. Por otro lado, en cuanto al $\mathrm{pH}$, no se observaron diferencias estadísticamente significativas entre las muestras $(6,48$ para $\mathrm{F} 1 ; 6,52$ para $\mathrm{F} 2$ y 6,60 para $\mathrm{Fc}$ ).

En relación al color, la formulación F1 $(40 \%$ de harina extrudida de ñame), fue la que presentó una mayor luminosidad con tendencia al color blanco $(\mathrm{L}=78)$, ya que para F2 (20\% de harina extrudida de ñame) y Fc (producto comercial), los valores de L fueron de 76 y 70 , respectivamente. Las diferencias existentes entre las muestras, se atribuyeron a la presencia de la harina extrudida de ñame en las formulaciones F1 y F2, la cual presentó un valor de L igual a 85, indicativo de

\section{TABLA 3}

\section{Características físicas de las mezclas en polvo para preparar bebidas instantáneas a base de la harina extrudida de ñame y de la harina extrudida de ñame}

\begin{tabular}{|c|c|c|c|c|}
\hline Características & F1 & F2 & Fc & $\mathbf{H N}$ \\
\hline aw* & $0,42 \pm 0,02^{\mathrm{a}}$ & $0,41 \pm 0,03^{\mathrm{a}}$ & $0,39 \pm 0,00^{\mathrm{a}}$ & $0,38 \pm 0,01^{\mathrm{a}}$ \\
\hline $\mathrm{pH}^{*}$ & $6,48 \pm 0,01^{\mathrm{a}}$ & $6,52 \pm 0,01^{\mathrm{a}}$ & $6,60 \pm 0,01^{\mathrm{a}}$ & $6,55 \pm 0,20^{\mathrm{a}}$ \\
\hline \multicolumn{5}{|l|}{ Color } \\
\hline L (luminosidad)* & $78,00 \pm 0,05^{\mathrm{b}}$ & $76,00 \pm 0,05^{\mathrm{c}}$ & $70,00 \pm 0,06^{c}$ & $85,00 \pm 0,04^{\mathrm{a}}$ \\
\hline Viscosidad (cps) & $1990,00 \pm 0,5^{\mathrm{a}}$ & $1802,00 \pm 0,01^{\mathrm{b}}$ & $1843,00 \pm 0,05^{\mathrm{b}}$ & $120 \pm 0,02^{\mathrm{c}}$ \\
\hline \multicolumn{5}{|c|}{$\begin{array}{l}\text { *Los valores se presentan como la media } \pm \text { desviación estándar } \\
\text { Los valores se presentan como la media } \pm \text { desviación estándar } \\
\text { F1: } 40 \% \text { harina extrudida de ñame, } 34,00 \% \text { leche entera en polvo, } 25,82 \% \text { azúcar en polvo, } 0,18 \% \text { vainillina en polvo } \\
\text { F2: } 20 \% \text { harina extrudida de ñame, } 54,02 \% \text { leche entera en polvo, } 25,80 \% \text { azúcar en polvo, } 0,18 \% \text { vainillina en polvo } \\
\text { Fc: producto análogo comercial } \\
\text { HN: harina extrudida de ñame. } \\
\text { Letras diferentes en una misma fila denotan diferencias estadísticamente significativas (P } \leq 0,05 \text { ). }\end{array}$} \\
\hline
\end{tabular}

\section{TABLA 4}

Evaluación sensorial por comparación múltiple entre rangos de las formulaciones de las mezclas en polvo para preparar bebidas instantáneas a base de la harina extrudida de ñame

$\begin{array}{lccr}\text { Atributos sensoriales } & \text { F1 } & \text { F2 } & \text { Fc } \\ \text { Sabor } & 3,70^{\mathrm{c}} & 4,25^{\mathrm{ab}} & 4,40^{\mathrm{a}} \\ \text { Color } & 4,01^{\mathrm{b}} & 4,55^{\mathrm{a}} & 4,30^{\mathrm{a}} \\ \text { Aroma } & 4,20^{\mathrm{b}} & 4,40^{\mathrm{a}} & 4,70^{\mathrm{a}} \\ \text { Consistencia } & 3,60^{\mathrm{b}} & 4,55^{\mathrm{a}} & 4,80^{\mathrm{a}}\end{array}$

Letras diferentes en una misma fila muestran diferencias estadísticamente significativas $(\mathrm{P} \leq 0,05)$

F1: $40 \%$ harina extrudida de ñame, $34,00 \%$ leche entera en polvo, $25,82 \%$ azúcar en polvo, $0,18 \%$ vainillina en polvo F2: $20 \%$ harina extrudida de ñame, $54,02 \%$ leche entera en polvo, $25,80 \%$ azúcar en polvo, $0,18 \%$ vainillina en polvo Fc: producto análogo comercial

$\mathrm{HN}$ : harina extrudida de ñame. 
una elevada tendencia al color blanco, que permanece en los productos formulados y que podría mantenerse a través del tiempo, debido a que la harina extrudida de ñame presenta ciertos compuestos antioxidantes que podrían evitar la aparición de cambios significativos en la coloración del producto (10).

Con relación a la viscosidad aparente, se encontraron diferencias significativas entre las muestras. El valor más alto se obtuvo para el polvo F1 (1990 cps), mientras que para el polvo F2 y el producto comercial $\mathrm{Fc}$, se encontraron valores iguales a 1802 y $1843 \mathrm{cps}$, respectivamente. Las diferencias existentes se atribuyeron al mayor porcentaje de sustitución con harina extrudida de ñame, a la incorporación de los sólidos que aporta la leche entera a la mezcla en polvo para bebida F1 y al aporte de las propiedades de la proteína contenida en esta, que contribuyeron al mayor desarrollo de la viscosidad. Los resultados obtenidos fueron superiores a los reportados por Arcila y Mendoza (26), en bebidas a base de amaranto, maíz y arroz (entre 1500 y 1650 cps), siendo estas diferencias posiblemente debidas a que los gránulos de almidón de ñame son de mayor tamaño y con fuerzas asociativas internas débiles, que contribuyen a una mayor capacidad de absorción de agua, y por tanto a una alta capacidad de formar pastas viscosas y espesas (29).

En la tabla 4 se señalan los resultados obtenidos en la evaluación sensorial de las formulaciones reconstituidas en agua, en la cual se determinó que existen diferencias estadísticamente significativas entre las muestras evaluadas. Se encontró que las bebidas obtenidas a partir de la mezcla en polvo con $20 \%$ de harina extrudida de ñame (F2) y del producto comercial $(\mathrm{Fc})$ fueron las más aceptadas en todos los parámetros evaluados, color, sabor, aroma y consistencia. Considerándose, que es posible que la mayor incorporación de la harina extrudida de ñame a la formulación F1, provocara el menor grado de aceptabilidad de la misma.

\section{CONCLUSIONES}

La mezcla en polvo para preparar bebidas instantáneas formulada a partir de un $20 \%$ de la harina extrudida de ñame, resultó ser la de mayor grado de aceptabilidad en los atributos sabor, color, aroma y consistencia, presentando características físicas y químicas similares a los productos análogos comerciales, e incluso con un mayor contenido de proteínas, fibra dietaría y polifenoles, que lo definen como un alimento de mejor valor nutricional.

\section{RESUMEN}

Los tubérculos de Dioscorea alata de la familia Dioscoreaceae, son rizomas cultivados principalmente en las regiones tropicales y subtropicales del mundo, que representan una fuente de alimentación energética para estas poblaciones, dado el alto contenido de almidón. En busca de incrementar y diversificar su consumo, se planteó estudiar la incorporación de la harina extrudida de ñame para la elaboración de una mezcla en polvo para preparar bebidas instantáneas, saborizada con vainilla. Se realizaron dos formulaciones a base de $20 \%$ y $40 \%$ de harina extrudida de ñame, evaluadas en función a la composición química, características físicas (actividad de agua, color, $\mathrm{pH}$ y viscosidad) y propiedades sensoriales. Los resultados indicaron que la incorporación de la harina extrudida de ñame, permitió incrementar el contenido de proteína y fibra dietaria, con valores de actividad de agua, $\mathrm{pH}$ y viscosidad similares al producto comercial tomado como referencia. La evaluación sensorial resultó de buena aceptación para las formulaciones estudiadas. En conclusión, la formulación a base de un $20 \%$ de la harina extrudida de ñame, permitió obtener un producto con propiedades químicas, físicas y sensoriales comparables al comercial, pero con propiedades antioxidantes, atribuidas a algunos polifenoles provenientes de la harina extrudida de ñame.

Palabras claves: Dioscorea alata, harina, polvos, bebidas instantáneas.

Dirigir la correspondencia a:

\section{Profesora}

Emperatriz Pachecho-Delahaye

Laboratorio de Bioquímica de Alimentos

Instituto de Química y Tecnología

Facultad de Agronomía

Universidad Central de Venezuela

Maracay, Estado Aragua,

Venezuela

Código Postal 2105

E-mail: olivier@movistar.net.ve

Agradecimientos: Los autores agradecen el financiamiento parcial del CDCH-UCV y la ayuda de la TSU Gloria Betancourt.

\section{BIBLIOGRAFÍA}

1. Jayakody L. Hoover R. Liu Q. Donner E. Studies on tuber starches. II. Molecular structure, composition and physicochemical properties of yam (Dioscorea spp.) starches in Sri Lanka. Carboh Polymers 2007; 10:1016-1023.

2. Pérez E. Pacheco Y. Características químicas, físicas y reológicas de la harina y el almidón nativo aislado de Ipomoea batatas. Acta Cient Venez 2005; 56: $12-20$.

3. Chou S. Chiang B. Chung Y. Chen P. Hsu C. Ef- 
fects of storage temperatures on the antioxidative activity and composition of yam. Food Chem 2006; 22(1): 618-623.

4. Araujo C. Rincón, A. Padilla, F. Caracterización del almidón nativo de Dioscorea bulbifera L. ALAN 2004; 54(2): 241-244.

5. Djerassi C. Drugs from third world plants: The Future Science 1992; 258: 203-204

6. Fu Y. Chen S. Lai Y. Centrifugation and foam fractionation effect on mucilage recovery from Dioscorea (yam) tuber. J Food Sci 2004; 69(9): 509-514.

7. Hou W. Lee M. Chen H. Liang W. Han C. Liu Y. Lin Y. Antioxidant activities of Dioscorin, the storage protein of yam (Dioscorea spp.) tuber. J. Agric Food Chem 2001; 49: 4956-4960.

8. Kaur C. Kapoor H. Antioxidant activity and total phenolic content of some Asian vegetables. Int J Food Sci Tech 2002; 37: 153-161.

9. Bhandari M. Kawabata J. Organic acid, phenolic content and antioxidant activity of wild yam (Dioscorea spp.) tubers of Nepal. Food Chem 2004; 88: 163-168.

10. Hsu C. Hurang S. Chen W. Weng Y. Tseng C. Qualities and antioxidant properties of bread as affected by the incorporation of yam flour in the formulation. Int J Food Sci Tech 2004; 39: 231-238.

11. Farhat I. Oguntona T. Neale R. Characterization of starches from West African yams. J Sci Food Agric 1999; 2105-2112.

12. Zaidul I. Norulaini N. Mohd A. Yamacuchi H. Noda T. RVA analysis of mixtures of wheat flour and potato, sweet potato, yam, and cassava starches. Carboh Polymers 2007; 69: 784-791.

13. Desrumaux A. Bouvier J. Burri J. Effect of free fatty acids addition on corn grits extrusion cooking. Cereal Chem 1999; 76(5): 699-704.

14. Kadan R. Pepperman A. Physicochemical properties of starch in extruded rice flours. Cereal Chem 2002; 79(4): 476-480.

15. Vasanthan T. Yeung J. Hoover R. Dextrinization of starch in barley flours with thermostable alphaamylase by extrusion cooking. Starch/Stârke 2001; 53: 616-622.

16. Shi X. Bemiller J. Effects of food gums on viscosities of starch suspensions during pasting. Carboh Polymers 2002; 50: 7-18.

17. García A. Pacheco E. Tovar J. Pérez E. 2007. Carac- terización fisicoquímica y funcional de la harina de arracacha (A. xanthorriza) para sopas instantáneas. CYTA, México 5(5):384-393.

18. Association of Official Agricultural Chemists. Official Methods of Analysis of the AOAC. 12 th ed. Washington, D.C., The Association, 1990.

19. Holm J. Björck I. Asp N. Sjöberg L. Starch availability in vitro and in vivo after flaking, steamcooking and popping of wheat. J Cereal Sci 1985; 3: 193-206.

20. Kaluza W. Mc.Grath R. Roberts T. Shoroder H. Separation of phenolics of sorghum bicolor. J Agric Food Chem 1980; 28: 1191-1196.

21. Pedrero D. Pangborn R. Evaluación sensorial de los alimentos. Métodos Analíticos. Editorial Alambra Mexicana. México. 1989.

22. Wittig de Penna E. Serrano L. Bunger A. Soto D. López L. Hernández N. Ruales J. Optimización de una formulación de espaguetis enriquecidos con fibra dietética y micronutrientes para el adulto mayor. ALAN 2002; 52(1): 91-99.

23. Blanco A. Tovar J. Fernández M. Caracterización nutricional de los carbohidratos y composición centesimal de raíces y tubérculos tropicales cocidos, cultivados en Costa Rica. ALAN 2004; 54(3): 322-327

24. Freitas R. Paula R. Feitosa J. Rocha S. Sierakoeski M. Amylose contents, rheological properties and gelatinization kinetics of yam (Dioscorea alata) and cassava (Manihot utilissima) starches. Carboh Polymers 2004; 55: 3-8.

25. Leonel M. Mischan M. Zambello S. Iaturo R. Duarte J. Efeitos de parâmetros de extrusão nas propiedades físicas de produtos expandidos de inhame. Cienc Tecnol Alim 2006; 26(2).

26. Arcila N. Mendoza Y. Elaboración de una bebida instantánea a base de semillas de amaranto (Amaranthus cruentus) y su uso potencial en la alimentación humana. Rev Fac Agron 2006; 23(1).

27. Pacheco E. Pérez R. Scnell M. Evaluación nutricional y sensorial de polvos para bebidas a base de papaya, plátano verde y salvado de arroz. Índice glucémico. INCI 2004; 29(1): 46-51.

28. Badui I. Química de Alimentos. Editorial Acribia. 1995.

29. Gebre T. Ababa A. Schmidt P. Some physico-chemical properties of Dioscorea starch from Ethiopia. Starch/Stärke 1998; 50: 241-246 